Oeuvres complètes de Christiaan Huygens publiées par la Sociêté Hollandaise des Sciences. Tome Quinzième. Observations Astronomiques. Système de Saturne. Travaux Astronomiques, 1658-1666. La Haye, Martinus Nijhoff, 1925. Pp. 622.

This fifteenth volume of the works of Huygens is a model of what can be done for the convenience of readers. A general table of contents (sommaire) containing twelve entries is supplemented by a fuller one (pièces et mémoires) covering eight pages. There are alphabetical indexes to persons and to subject matter. Numerous footnotes contain explanations and cross references. The longer documents appear in the original Latin and in French translation. Facsimile reproductions of parts of manuscripts and original drawings add to the interest of the book. Introductions to the various parts contain information in great detail. Among the mathematical notations of interest are the Cartesian sign of equality (the sign for taurus turned so that the opening is on the left) and the "scratch method" used in extracting square roots.

Huygens' early astronomical observations relate mainly to Saturn, Jupiter and their companions. Huygens and his brother Constantine constructed their own telescopes. The first one, 12 feet in length, promptly revealed a new satellite of Saturn. This instrument and a 23 foot telescope furnished data to solve the enigma relating to the varying appearance of Saturn. Galileo had seen it threefold. Hevelius saw it in three parts. Fontana made drawings widely distorted. Huygens in 1655 drew Saturn as a sphere with two handles or arms on opposite sides and extending in opposite directions. When Saturn was near the sun, in January, 1656, the arms disappeared. Nevertheless, Huygens had a hypothesis which he published in 1656, disguised as an anagram: aaaaaacccccdeeeeghiiiiiiilll1 mmnnnnnnnnnooooppqrrstttttuuuuu. Later in 1656, the handles reappeared and gradually changed so as to confirm his theory. In 1657 and 1658 , the rings became somewhat plainer, and in 1659 he published his Systema Saturnium in which he explained his anagram as signifying a Latin sentence which is in translation: "It is surrounded by a slender flat ring, everywhere distant from the surface, and inclined to the ecliptic."

FloRIAN CAJORI

Einfiihrung in die Analytische Geometrie. By A. Schoenflies. Berlin, Julius Springer, 1925. $\mathrm{x}+304 \mathrm{pp}$.

This book is volume XXI of the series Die Grundlehren der mathematischen Wissenschaften in Einzeldarstellungen, and in general excellence of treatment is in keeping with the other volumes of the series. The author states in the preface that there is perhaps a greater contrast between the methods of elementary and advanced analytic geometry than those of any other branch of mathematics, and this book will be very helpful to the student who is in this transition stage in his study of geometry.

For the purpose of this review the book will be divided arbitrarily into four parts including respectively Chapters I-V, VI-VIII, IX-XII, and XIII-XVII inclusive; the first two of these parts being approximately 
50 pages each and the last two 80 pages each. To this is added an appendix and index of 40 pages.

The first five chapters include the fundamental elementary topics which are studied in a first course in analytic geometry. These are developed in the familiar non-homogeneous analytical language but from a broader and more general point of view than the method of the ordinary text book. After explaining various coordinate systems the equations of the conics are derived in both the cartesian and polar systems together with the parametric equations of the circle and ellipse. The distance of a point from a line is found by means of projections. This relation and the transformation of coordinates precede the chapter on the straight line in which the importance of the Hesse normal form is shown and which includes a good treatment of the theory of two lines and of three lines.

In Chapters VI-VIII inclusive the fundamental framework of modern analytic geometry is constructed. The projective method predominates as this gives the most natural approach to homogeneous coordinates. The author develops this part in logical order and with great clearness. It includes line coordinates, duality, the complete 4-point and 4-line, the theorems of Desargues (perspective triangles) and Pascal, double ratio, and the theory of harmonic pairs. Linear substitution is shown to be a projective relation; quadratic involutions are discussed; and the conic is obtained as a locus or envelope of corresponding projective forms. Homogeneous coordinates are developed first from cartesian point and line coordinates, then linear projective coordinates are introduced. Finally the general plane homogeneous coordinates are defined by means of a triangle of reference, and the ratios of these coordinates are shown to be given by means of a double ratio as soon as the unit point is fixed.

Chapters IX-XII inclusive deal principally with a more extensive treatment of the conics, the general equation of the second degree, and collineations in the plane. Orthogonal pencils of circles, circular points and minimal lines, and transformation by reciprocal radii are included in the chapter on the circle. In the following chapters are discussed confocal conics, conjugate diameters, invariants, polar systems and involutions, the pencil of conics, collinear and reciprocal transformations, and the theorems of Pascal and Brianchon.

The last five chapters are devoted to the geometry of three dimensions following a procedure analogous to that used in the plane. Coordinate systems, dual elements and loci, collineations in space, a discussion of the quadric surfaces both with non-homogeneous and with the general homogeneous coordinates, and the polar system are the principal subjects considered. Useful related algebraic topics are given a supplementary treatment in the appendix, including determinants, linear equations, and transformations. The book ends with a selected list of 115 exercises and problems.

After introducing the homogeneous coordinate systems it seems unfortunate that the author did not make more extensive use of them. Had the equation of the circle, for instance, been given with an inscribed triangle of reference it would have opened to the student a most attractive 
method for investigating the geometry of the circle and triangle. However, the arrangement of the material is logical and successive topics are developed in a masterly fashion. It should be kept in mind that the author has attempted to fulfill not only the formal requirements of the Wissenschaften but also the demands of the student in geometry both as to the selection and arrangement of material. In this sense the book is a compromise; to quote from the preface "ein einführendes Lehrbuch soll in erster Linie ein Lernbuch sein." A few minor errors in type were observed but these do not detract from the merits of the book. It is a welcome addition to our libraries and will be a valuable reference for students in their first course in modern analytic geometry.

\section{J. I. TRACEY}

La Logique des Mathématiques. By Stanislas Zaremba. Mémorial des Sciences Mathématiques, No. 15. Paris, Gauthier-Villars, 1926.52 pp.

In this number of the Mémorial des Sciences Mathématiques, Professor Zaremba, of the University of Cracow, views logic as a "theory of deductive demonstration" and sketches a "logistic" by means of which a "complete" mathematical demonstration can be effected. In the course of his exposition, in which he "carefully refrains from engaging in psychologic and philosophic speculations," the author formulates fundamental problems in the logic of mathematics still awaiting solution, and gives an explanation of the paradoxes in aggregate theory based on a distinction between the notions class and category. The text is followed by a good-sized bibliography.

The author excludes from his logic the logic of classes and the logic of relations - theories that "really should not enter into the domain of a general theory of demonstration." His logic is a logic of propositions consisting (1) of a theory of "propositional identities" and (2) of a theory of propositions of logic of the "second kind." The first of these seems to be the theory of "elementary" propositions of Whitehead and Russell's Principia Mathematica, whose "theory of deduction" is "the most complete exposition of the theory of propositional identities." The second theory is presumably that of "apparent variables" of the Principia. The author, however, does not accept Russel's "theory of types," which has brought into logic "numerous obscurities and extreme complications."

B. A. Bernstein

Esquisse d'Ensemble de la Nomographie. (Mémorial desSciences Mathématiques, No. 4.) By Maurice d'Ocagne. Paris, Gauthier-Villars, 1925. 68 pp.

This is one of the little volumes on interesting mathematical subjects now being published under the direction of Professor Henri Villat as Mémorial des Sciences Mathématiques. It covers in four chapters of twenty-eight sections most of what is known of nomographic theory. To the advanced student the treatment offers interest and charm for the presentation has authority, brevity and elegance. The engineer or physi- 